\title{
Buoyant Convection in an Inclined Enclosure
}

\author{
R. G. REHM, H. R. BAUIM, D. W. LOZIER, H. TANG and J. SIMS \\ National Institute of Standards and Technology \\ Gaithersburg, Maryland 20899, USA
}

\begin{abstract}
Equations for a Boussinesq model describing transient buoyant convection driven by a heat source in a rectangular enclosure are presented and solved by finite difference methods. Gravity is allowed to have an arbitrary direction relative to the enclosure so that the enclosure is inclined to horizontal. Computational results for three-dimensional dissipation-free flows and for two-dimensional flows with and without dissipation are presented. The hydrodynamics is based directly on the time-dependent Euler or Navier-Stokes equations. No turbulence model or other empirical parameters are introduced. The previous algorithms had been verified by comparisons with exact solutions to the equations in simple, special cases, and overall predictions of the model when the viscosity and thermal conductivity are zero have been compared with experimental results. The use of Lagrangian particle tracking allows one to visualize the flow patterns. The effects of a fire-induced flow in a corridor, and a stair well (or escalator) are examined.
\end{abstract}

Key Words: Boussinesq; Buoyant Convection; Computational Fluid Dynamics; Enclosure Fires; Finite Difference Methods; Fire-Induced Flows; Transient Flows

\section{Introduction}

Fires in buildings involve the transport of heat and mass by gravity-induced or buoyant convection. Generally, this convection occurs in rectangular enclosures where the direction of gravity is parallel to the surfaces of the enclosure, the walls. However, under certain circumstances, such as a fire in a stair well or an escalator, the enclosure may be sloped relative to gravity. A very important example of a fire in a sloped enclosure was the devastating fire in the King's Cross underground station in England in 1987, where there was significant loss of life as well as property damage. Numerical simulation of this fire [1] uncovered an unexpected phenomenon which caused a very rapid spread of the fire and led to much of the devastation. This phenomenon was termed "the trench effect," and caused some controversy during investigations of the King's Cross fire in England. The phenomenon was ultimately confirmed by experiments and additional simulation [2], but transient aspects of the fire simulation 
are still of interest. It is the purpose of this paper to report an examination of this phenomenon using a very different mathematical and computational model than those used in the cited references; our model had been especially constructed to examine time-dependent buoyant convection and has been used to examine fire-driven flows.

The authors have previously published descriptions of the mathematical model and algorithm which they developed for computation of the buoyant convection induced by a fire evolving in a room [3], [4], [5], [6]. The model is for a dissipation free, thermally expandable fluid, i.e., one for which density and temperature variations can be large, but pressure variations are small [3], and for a Boussinesq model, one in which the density variations are also small. All of the work reported in this paper is for the Boussinesq model only. For the three-dimensional computations, the dissipationfree model described in the papers cited above is used. This model is used because one is unable to resolve both large-scale motions associated with room-scale buoyant convection and motions at the dissipative length scale with computational resources available. However, with the revolution in computational capability currently taking place, an ability to resolve both small scale and large scale features of the flow is now possible in two dimensions. In this document the authors report computational results obtained from a generalization of their model and algorithm, in two dimensions, to include viscous dissipation and thermal conduction. We present a brief description of the algorithm. When the model is restricted to two dimensions, very high resolution computations can be performed, and these computations allow us to resolve both largescale buoyant convection and small scale dissipation for Reynolds numbers of interest for enclosure fires. The two- and three-dimensional results are compared because the "trench effect" phenomenon is essentially two dimensional we believe. First, we present the model of buoyant convection in Section 2, then a description of the finite difference equations used to solve the model is presented in Section 3, and finally, results, together with interpretation of the physical phenomena observed in the nurnerical computations are described in Section 4.

\section{Hydrodynamic Model}

Traditionally, two approaches to the computation of fire-induced buoyant convection have been reported: direct integration of the Navier-Stokes equations using molecular values for viscosity and thermal conductivity or integration of these equations using a turbulent viscosity and conductivity to account for fluctuations occurring at the large Reynolds numbers of practical interest. The former approach, although most desirable, is not practically feasible in three dimensions with todays computers, but has become practical in two dimensions. Alternately, the use of a turbulence model in the equations introduces functional forms and empirical constants which do not have a fundamental theoretical basis at this time. Direct simulation of the Navier Stokes equations at Reynolds numbers of practical interest for fire-driven flows is now possible in two dimensions, and these simulations are the subject of this paper.

We consider a Boussinesq fluid with constant or zero values of the viscosity and thermal conductivity in a rectangular enclosure driven by a prescribed heat source. The essence of the buoyant convection model is described as follows. We start with the Navier-Stokes equations for a Boussinesq fluid and combine them as described in 
[3]. The nondimensional equations can be written as follows:

$$
\begin{aligned}
\frac{\partial \rho}{\partial t}+\vec{u} \cdot \nabla \rho & =-\tilde{Q}+\frac{1}{\operatorname{Re} \operatorname{Pr}} \nabla^{2} \rho \\
\frac{\partial \vec{u}}{\partial t}+\vec{F}+\nabla p-\rho \hat{g} & =\frac{1}{\operatorname{Re}} \nabla^{2} \vec{u} \\
\nabla^{2} p & =f
\end{aligned}
$$

where

$$
\begin{aligned}
\vec{F} & =-\vec{u} \times \omega+\nabla\left(u^{2} / 2\right) \\
\vec{\omega} & =\nabla \times \vec{u} \\
f & =-\nabla \cdot(\vec{F}+\rho \hat{g})
\end{aligned}
$$

Here, all symbols have their usual fluid dynamical meaning: $\rho$ is density, $\vec{u}$ is the velocity, $p$ is pressure, $\hat{g}$ is the vector describing the direction of gravity, Re is the Reynolds number, $\operatorname{Pr}$ is the Prandtl number, $t$ is time and $\tilde{Q}$ is proportional to the spatially and temporarily prescribed heat source.

All symbols have their meanings in dimensionless form. If we let all variables with tildes denote dimensional quantities and those without denote dimensionless ones, then the dimensional and dimensionless variables are related as follows: $\tilde{\vec{u}}=U \vec{u}, \tilde{p}=$ $\rho_{0} U^{2} p, \tilde{\rho}=\rho_{0}\left(U^{2} / g H\right) \rho, \tilde{\vec{x}}=H \vec{x}, \tilde{t}=(H / U) t$, where $U$ is the velocity scale, $H$ the height of the enclosure and $\rho_{0}$ is the ambient density. To define the velocity scale, we introduce the following quantities. $Q_{0}$ is the rate of heat addition in the three dimensional case (energy per time), $q_{0}$ is the heat addition per unit length in the two-dimensional case (energy per length per time), $g$ the acceleration of gravity, $T_{0}$ is the ambient temperature, and $C_{p}$ is the constant-pressure specific heat. Then, in the three-dimensional and two-dimensional cases respectively,

$$
U=\left(Q_{0} g /\left(\rho_{0} C_{p} T_{0} H\right)\right)^{1 / 3}=\left(q_{0} g /\left(\rho_{0} C_{p} T_{0}\right)\right)^{1 / 3}
$$

The Reynolds and the Prandtl numbers are defined as follows: $\operatorname{Re}=U H / \nu$ and $\operatorname{Pr}=\mu C_{p} / k$, where $\mu$ is the viscosity, $k$ is the thermal conductivity and $\nu=\mu / \rho_{0}$ is the kinematic viscosity. See [4] and [7] for more information on the scaling.

Boundary conditions used for these equations are that there be no inflow or outflow at boundaries, that either there be a no-slip or free-slip condition at boundary walls and that the walls are either adiabatic or kept at a constant temperature. The initial conditions are that the fluid is quiescent.

\section{Numerical Methods}

Equations (1) are either a mixed parabolic/elliptic system of partial differential equations, or, if $\nu=k=0$, a mixed hyperbolic/elliptic system; i.e., the equations for the density and for the velocity components are parabolic (hyperbolic), whereas that for the pressure is elliptic. The incompressible equations of hydrodynamics are well known to have this mixed character. 
When $\nu=k=0$, there is no dissipation in these equations, and it is important not to introduce any through the numerical scheme. Analytical studies of the ability of several candidate finite difference schemes to calculate internal gravity waves [8] led to the conclusion that methods of second order accuracy in space and time would be necessary; the scheme chosen is dispersive, but not dissipative. All time derivatives are replaced by central differences over twice the time step size (a leap-frog scheme). Other terms in the evolution equations (the first two of Eqs. (1)) are in general evaluated at the mid level of the three level leap-frog scheme. An exception arises in the vertical momentum equation, where in the buoyancy term the density is taken to be the average of the density at the top and bottom of the three level scheme; this semi-implicit nature of the scheme is required for stability (see [8], [4] and [6] for details). Small-scale numerical fluctuations arising during computation are removed by stopping periodically the calculations, smoothing and restarting [12]. When viscosity and thermal conductivity are present, a second-order leap-frog (Fromm) method or a lagged-diffusion scheme have been used for temporal updating, causing no additional stability limitations and only a straight-forward generalization of the algorithm discussed in the references cited above.

The spatial grid is taken to be uniform in each of the two or three directions, although the mesh length may be different in each direction. Within each mesh cell, a parallelepiped, vector components are evaluated at the faces and scalar quantities at the center of the cell. The staggered grid permits central differences to second order accuracy for all linear operations. The nonlinear terms must be considered separately. The density evolution equation in continuous form is the mass conservation equation minus the expression for the velocity divergence. Each of these two equations is approximated by central differences and then subtracted. The density at all faces is approximated by the mean of the density at the centers of adjacent cells. This procedure ensures global mass conservation as well as second order accuracy.

The momentum equation is differenced in the vector invariant form. This ensures nonlinear stability and complete compatibility between the "primitive variable" formulation presented here and a vorticity, stream-function formulation (in the twodimensional case), see [10] for details. When dissipation is absent, the finite difference scheme for the momentum equations is presented in detail in [4] and [10].

The pressure equation is the discretized version of the time derivative of the incompressibility (zero velocity divergence) condition, using the central difference approximation to the divergence of the velocity and with the time difference of the velocity replaced using the discretized momentum equations. Mathematically, the calculation of the pressure requires the solution of an elliptic partial differential equation. The linear algebraic system arising from its discretization has constant coefficients and can be solved by a fast direct method, see [6] for details. The solution to the pressure equation constitutes the bulk of the numerical computation since the density and the velocity are updated explicitly once the pressure gradients are known.

Finally, stability of the computational scheme imposes a limit on the time step size relative to the spatial mesh sizes, [4] and [8]. Also, accuracy of the computation is an important consideration, which has been examined in [8], [4] and [6], and verification that the numerical methods solve the partial differential equations, at least in special cases, has been addressed in these references. In addition, the basic features, such as the plume rise-time in a uniform density environment, have been compared with 
experimental results to verify the predictive capability for the dissipation-free model, [7]. With such careful consideration of the basic Euler model and numerical methodology, we have confidence in the predictions of the large-scale phenomena made by these computations, and feel justified in interpreting large-scale physical features arising in them.

The computations reported here were performed on the N.I.S.T. Convex C120 obtained for computational combustion. Each computation requires between eight and thirty CPU hours depending upon the conditions of the computation. The most satisfactory means for visualization of the computational output, we have found, is to display the locations of Lagrangian particles introduced into and convected by the flow field. These displays can be dynamically performed using color to show the temperature field for example, and have been implemented on Silicon Graphics Personal Irises which are also part of the computational combustion facility. Several frames taken from the screen of the SGI workstations displaying these particles are presented, and the physical interpretation of the results are discussed. It should be emphasized that still pictures cannot convey the sense of movement obtained when the viewer observes the dynamical display on the graphics device. Similarly, depth perception for these three-dimensional calculations and Lagrangian particle plots, which is provided on the graphics device, is diminished in the static figures. Finally, color adds immeasurably to the fluid-dynamical interpretation.

\section{Results}

Computations of many cases for both two-dimensional and three-dimensional enclosures tilted at a variety of angles have been performed. In the three-dimensional cases and in the 2-D case shown in Figure 3, calculations were performed using the Euler equations (but with periodic smoothing) as described in earlier papers of the authors [3]-[10], [12]. In the two-dimensional case, very high resolution computations have been run, using over one quarter million cells in many cases. In most of the 2-D calculations, the Navier-Stokes (N-S) equations were integrated. The Reynolds number is limited by the resolution of the computations; it must be less than, but can be of the order of the number of grid cells. There are two effects of dissipation: there is a smoothing of the flow by viscosity and conduction, which occurs in the interior of the flow field, and there are boundary-layer effects, which can generate small-scale structures at the boundary. In the 2-D N-S computations, dissipative smoothing occurs while changes produced by altering boundary conditions have been examined. The results shown in Figure 3, where the Euler equations have been integrated, are virtually indistinguishable from results computed from the $\mathrm{N}-\mathrm{S}$ equations with large Reynolds numbers when adiabatic, free-slip BS are imposed. In the following subsections, several results are presented and discussed which show the effects on buoyant convection of inclining an enclosure relative to horizontal.

\subsection{3-D Horizontal Corridor}

In Figure 1, we present one frame from a sequence generated by the computation of a corridor (four times as long as the height or width of the corridor) flow induced by 
a heat source (fire) located at a position on the floor one quarter of the length of the corridor and one quarter the width from the end wall and side wall respectively. This calculation shows the full three dimensional nature of the flow. The viewing orientation is from the end of the corridor opposite to where the fire is located and from the wall closest to the fire. The particles rise in a buoyant plume above the fire, hit the ceiling and spread across the width of the corridor and down the side walls, forming a heated gas wedge that overlies the cool ambient gases in the corridor. This heated wedge of gases propagates down the corridor in a gravity current. The undulatory character of the upper layer, as shown in Figure 1, is due to the three-dimensional nature of the flow, a sloshing of the upper layer as internal waves are excited as the corridor is filled by heated gases. Finally, the gravity current hits the back wall, is reflected and returns toward the heat source (not shown here).

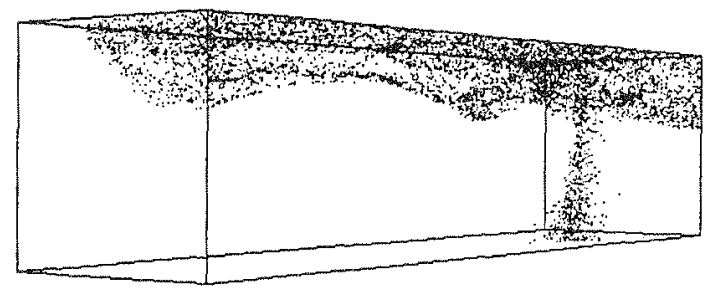

Figure 1 The dimensionless time shown is 4.5 units.

\subsection{3-D Corridor Inclined 35 Degrees}

Figure 2 shows two frames of a corridor flow generated as described for Figure 1 with the heat source similarly placed. In this case, however, the corridor is inclined 35 degrees with respect to horizontal; the end of the corridor opposite to the heat source is higher. The viewing angle is from the side of the corridor, and the time associated with each of the two frames is given in the figure caption. The initial buoyant plume begins to rise above the heat source at a 35 degree angle (opposite to the direction of gravity, which is pointed downward). The plume hits the ceiling and spreads both laterally toward the side walls and up the corridor toward the far wall. Because the corridor is inclined up toward the far end, the heated gases are accelerated toward the back wall as shown in the first frame. At similar times, the heated gases progress much further toward the far end than in the horizontal-corridor case. The high end, or end away from the fire, is filled by the smoke and hot gases, with vigorous mixing taking place in the filling volume as shown in the second frame of Figure 2. These results are essentially as expected for buoyant convection from a heat source in the inclined corridor.

\subsection{Corridor at 35 Degrees - 3-D and 2-D Compared}

In each of the calculations shown in the preceding figures, there are many interesting fluid-dynamical phenomena occurring; close examination of the results on the dynamical display on the workstation shows these phenomena. However, we will focus on only 
one phenomenon here, a very curious effect, which we believe is the "trench effect" uncovered and discussed in the Kings Cross investigation.
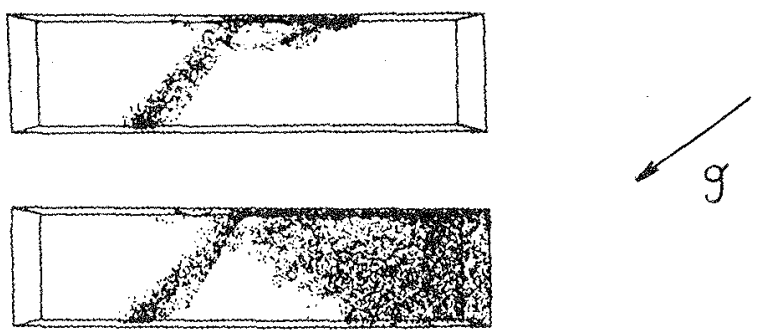

Figure 2 A three-dimensional flow in a corridor inclined at 35 degrees; the flow is shown at two dimensionless times, 2.25 and 8.0 .
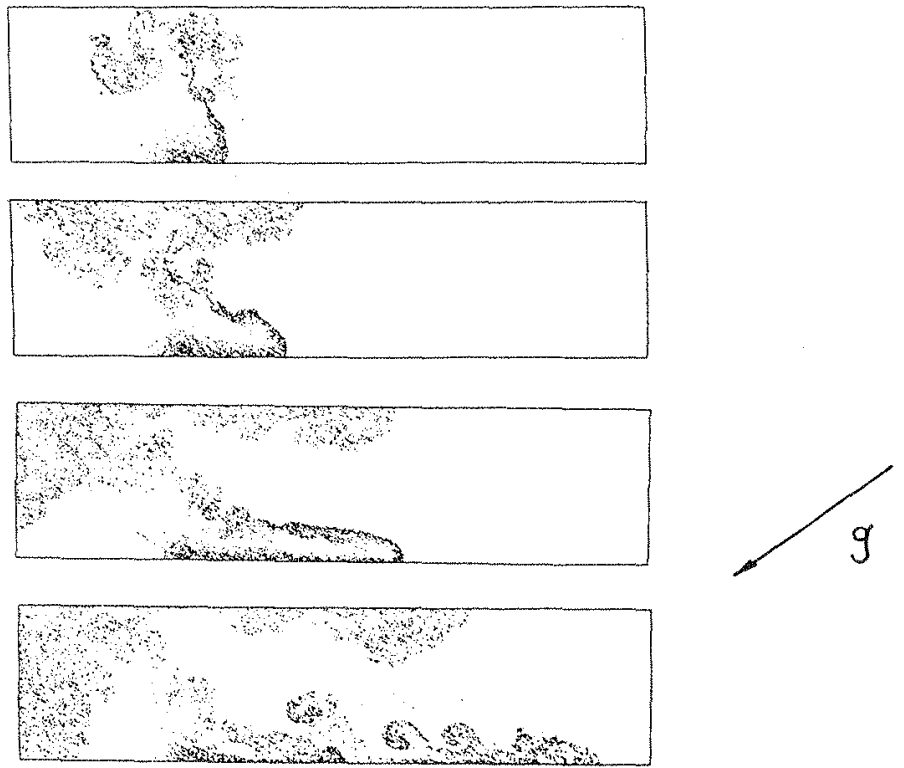

Figure $3 \mathrm{~A}$ two-dimensional Euler flow computation in a 35-degree inclined corridor The dimensionless times are 5, 7, 9 and 11 . 
In Figure 3 a collage of four frames from a high resolution 2-D computation of the Euler equations is shown. The resolution of all of the 3-D computations shown in the preceding figures is $144 \times 36 \times 36$ (186,624 grid cells) in the $4 \times 1 \times 1$ corridor; in Figure 3, the resolution is $1024 \times 256(262,144$ grid cells) in the $4 \times 1$ corridor, a factor of 7 more resolution in each direction. Because the computation shown in Figure 3 is a two dimensional version of the code used to compute the results shown in the preceding figures, the nondimensionalization is different; therefore, the dimensionless times ascribed to the various frames in Figure 3 cannot be compared with the times shown in the preceding figures. In this figure, the plume rises, but is bent back toward the back wall. After the hot gases hit the ceiling, they progress both toward the back wall and up the ceiling toward the high end. However, the hot gases leaving the heat source are pinned along the floor and form a hot gas jet which progresses up along the floor, shedding hot gases near its front; this phenomenon we interpret as the "trench effect".

To confirm this unexpected behavior, we performed a 3-D calculation identical to that shown in Figure 2 except that the heat source was spread across the width of the corridor. In the previous 3-D calculation, the heat source, although spatially distributed, was confined to a small region around its center; the center was located along the floor with its intensity decreasing in an axially symmetric Gaussian fashion with a half-width of one tenth of the width of the corridor. In the 3-D calculation with a 2-D source, the half-width of the Gaussian in the direction across the corridor was increased by three orders of magnitude so that there was essentially no decrease in source intensity across the corridor width. The computation qualitatively showed the same behavior as that shown in Figure 3 ; hence, we confirmed that the "trench effect" is essentially a 2-D effect, requiring that the heat source be spread across the corridor.

\subsection{Corridor at 35 Degrees - Effects of Boundary Conditions}

All of the results shown above were computed from a model in which there is no dissipation, i.e., no viscosity or thermal conductivity to diffuse (dissipate) momentum or heat. In the following figures, results will be shown from computations of the model in which viscous and thermal diffusion are included, i.e., the Navier Stokes equations are integrated as described earlier. All computations shown were performed on a $1024 \times 256$ grid, for a Reynolds number of $0.5 \times 10^{5}$; this grid allows stable and accurate computations with resolution of the boundary layer at the Reynolds number used. Since previous computations were dissipation free, the boundary conditions used were those appropriate to the Euler equations, namely, no normal moment um and heat fluxes at the boundaries.

To determine the effects of changing boundary conditions (B.C.), we performed a series of two-dimensional computations with varying B.C. The B.C. selected were adiabatic, no-slip (ans), cold-wall, free-slip (cfs), cold-wall, no-slip (cns), and a base line computation with adiabatic, free-slip (afs). The degree of realism of the various B.C. for any given physical situation (e.g., fire-driven flows, or salt-water driven flows in fresh water) can be debated, but the comparison between the various cases is of interest because one can imagine scenarios in which each of the B.C. is appropriate. Figure 4 shows a comparison between the computations with the different boundary 
conditions. It is composed of a frame at the same dimensionless time (5.0) from each of the four computations. The lower left plot is the base line computation with adiabatic, free-slip (afs) boundary conditions; the lower right plot shows adiabatic, no-slip (ans) boundary conditions; the upper left cold boundary, free-slip (cfs); and the upper right shows cold-wall, no-slip (cns) boundary conditions. Figure 5 shows a simliar comparison but at a later dimensionless time (9.0). Note that the plots in the lower left corner, the base-line ones, exhibit vividly the "trench effect", whereas, those in the upper right exhibit a more traditional plume rise above the heat source. The other sets of boundary conditions show intermediate behavior, with the hot gases clinging to the floor of the inclined corridor, interrupted by periodic separation of the hot gases from the floor into the enclosure. We interpret these results as indicating that the buoyant gases will cling to the floor when a fire spreads across the width of an inclined corridor (the trench effect) when boundary layer effects do not disrupt the weak buoyant flows. However, either vorticity generated along the floor by the boundary layer or cooling of the flow by a cold wall tend to break up the "trench effect".

The computations described here are induced by a heat source prescribed as a function of space and time with no combustion model. A very interesting extension of the model would be to include the effects of fire spread, which would affect the flow behavior determined above. This extension can be accomplished by including an appropriate combustion model [11]. We hope in the future to make such an extension.

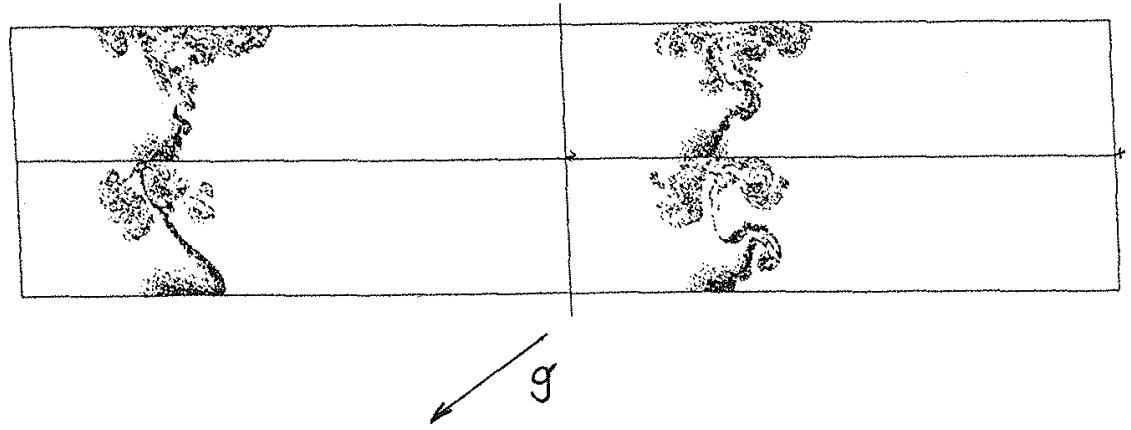

Figure $4 \mathrm{~A}$ composite of frames from four calculations at dimensionless time, 5.0. The computations are similar to those described in Figure 3, except with dissipation ( $\left.R e=2 \times 10^{5}\right)$. The lower left frame is for adiabatic, free-slip $\mathrm{BC}$, lower right for adiabatic, no-slip, upper left for cold-wall free-slip, and upper right for cold-wall noslip BC. 


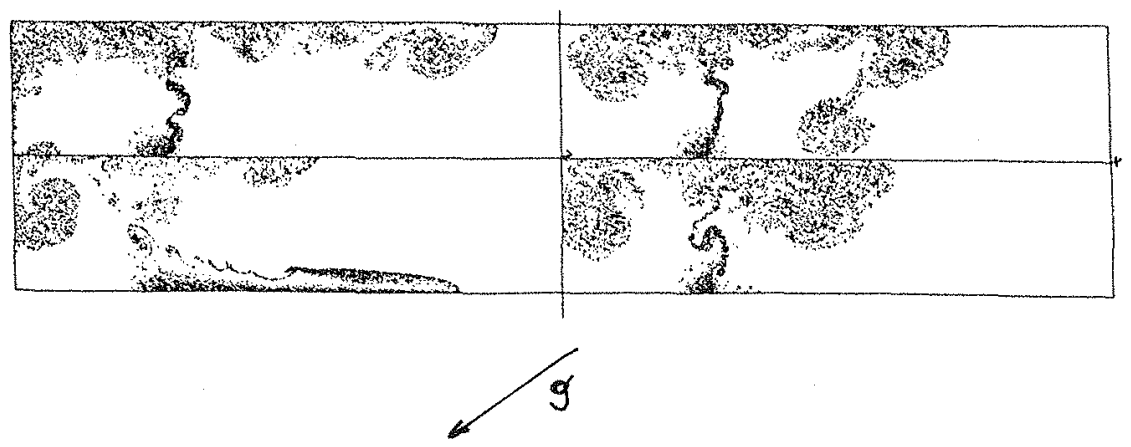

Figure $5 \mathrm{~A}$ composite of frames at dimensionless time 9.0 from the four calculations described in Figure 4.

\section{References}

[1] Simcox, S., Wilkes, N.S., and Jones, I.P. "Fire at King's Cross Underground Station, 18th November 1987: Numerical Simulation of the Buoyant Flow and Heat Transfer", Harwell Report AERE-G 4677, May 1988.

[2] Cox, G., Chitty, R. and Kumar, S., "Fire Modeling and the King's Cross Fire Investigation", Letter to the Editor, Fire Safety Journal 15, pp 103-106, 1989.

[3] Rehm, R.G. and H.R. Baum, "The Equations of Motion for Thermally Driven, Buoyant Flows", Journal of Research of the NBS, Vol. 83, pp 297-308, May-June 1978.

[4] Baum, H.R., R.G. Rehm, P.D. Barnett and D.M. Corley, "Finite Difference Calculations of Buoyant Convection in an Enclosure", SIAM J. Sci. Stat. Computing, Vol. 4, pp 117-135, March 1983.

[5] Rehm, R.G., Baum, H.R., Lozier, D.W. and Corley, D.M., "A Model of ThreeDimensional Buoyant Convection Induced by a Room Fire", First National Fluid Dynamics Congress, joint conference sponsored by the A.I.A.A., A.S.M.E., A.P.S. and S.I.A.M., July 24-28, 1988, Cincinnati, Ohio.

[6] Baum, H.R. and R.G. Rehm, "Calculations of Three-Dimensional Buoyant Plumes in Enclosures", Combustion Science and Technology, Vol. 40, pp 55-77, Gordon and Breach Science Publishers, 1984.

[7] Baum, H.R., Rehm, R.G. and Mulholland, G.W., "Computation of Fire Induced Flow and Smoke Coagulation", Nineteenth Symposium (International) on Combustion/ The Combustion Institute, pp 921-931, Pittsburgh, PA, 1982. 
[8] Baum, H.R. and Rehm, R.G., Finite Difference Solutions for Internal Waves in Enclosures, SIAM J. Sci. Stat. Comput., Vol. 5, No. 4, pp. 958-977 (1984).

[9] Baum, H.R. and Rehm, R.G., "Transient Combustion in a Turbulent Fire", 9th Joint Meeting of the U.S. Japan Natural Resources (UJNR) Panel on Fire Research and Safety, Boston, MA, May, 1987.

[10] Rehm, R.G., P.D. Barnett, H.R. Baum and D.M. Corley, "Finite Difference Calculations Of Buoyant Convection in an Enclosure: Verification of the Nonlinear Algorithm", Applied Numerical Mathematics, Vol.1, pp 515-529, North-Holland, 1985 .

[11] Baum, H.R., Rehm, R.G. and Gore, J.P., "Transient Combustion in a Turbulent Eddy", Twenty Third International Symposium on Combustion, to appear.

[12] Rehm, R.G., Baum, H.R. and Barnett, P.D., "Buoyant Convection Computed in a Vorticity, Stream-Function Formulation," Journal of Research of the NBS, Vol. 87, pp 165-185, March-A pril 1982. 
